\title{
Isolation and Identification of Shoot-Tip Associated Endophytic Bacteria from Banana cv. Grand Naine and Testing for Antagonistic Activity against Fusarium oxysporum f. sp. cubense
}

\author{
Aparna Chandra Sekhar, Pious Thomas* \\ Endophytic and Molecular Microbiology Laboratory, Division of Biotechnology, ICAR-Indian Institute of \\ Horticultural Research (IIHR), Bangalore, India \\ Email: ${ }^{*}$ pioust@gmail.com, ${ }^{*}$ pioust@iihr.ernet.in
}

Received 4 March 2015; accepted 10 April 2015; published 14 April 2015

Copyright (C) 2015 by authors and Scientific Research Publishing Inc. This work is licensed under the Creative Commons Attribution International License (CC BY). http://creativecommons.org/licenses/by/4.0/

(c) () Op Open Access

\section{Abstract}

Endophytic bacteria colonizing the shoot-tips of banana cv. Grand Naine were isolated and tested for the antagonistic activity against the Panama wilt pathogen Fusarium oxysporum f. sp. cubense (Foc). Pre-isolation, the suckers were given extensive disinfection treatments and the homogenate from the excised shoot-tip portion was plated on nutrient agar (NA) and trypticase soy agar (TSA). This yielded altogether 47 isolates: 26 on NA and 21 on TSA, respectively, from the 10 suckers collected during August to February. The number of bacterial isolates obtained per sucker varied from one to 15 based on colony characteristics registering up to 10 distinct species per shoot-tip based on $16 \mathrm{~S}$ rRNA sequence analysis. The 47 isolates belonged to 19 genera and 25 species under the phylogenetic classes of Actinobacteria, $\alpha$ - and $\gamma$-Proteobacteria and Firmicutes. Actinobacteria constituted the predominant phylum (55\% isolates) with the constituent genera of Arthrobacter, Brevibacterium, Corynebacterium, Curtobacterium, Kocuria, Kytococcus, Micrococcus, Naumanella, Rothia and Tessaracoccus spp. and an unidentified isolate belonging to the family Frankiaceae. Proteobacteria constituted the second major phylum (Brevundimonas, Enterobacter, Klebsiella, Pseudomonas, Serratia and Sphingomonas spp.) followed by Firmicutes (Bacillus and Staphylococcus spp.). Antagonistic activity of the endophytes against Foc was tested through agar plate assays (pit and spot applications on fungal lawn) employing potato dextrose agar and NA. Endophytic Pseudomonas aeruginosa (isolate GNS.13.2a) which was associated with a single sucker showed significant growth inhibition effect on Foc while Klebsiella variicola (GNS.13.3a) and Enterobacter

\footnotetext{
"Corresponding author.
}

How to cite this paper: Sekhar, A.C. and Thomas, P. (2015) Isolation and Identification of Shoot-Tip Associated Endophytic Bacteria from Banana cv. Grand Naine and Testing for Antagonistic Activity against Fusarium oxysporum f. sp. cubense. American Journal of Plant Sciences, 6, 943-954. http://dx.doi.org/10.4236/ajps.2015.67101 
cloacae (GNS13.4a) exhibited moderate inhibition. The study brings out considerable sucker to sucker variation in the associated cultivable endophytic bacteria in "Grand Naine" banana and identifies a few bacterial endophytes with biocontrol potential against the devastating Foc pathogen.

\title{
Keywords
}

\author{
Bacterial Endophytes, Bacterial-Fungal Antagonism, Biological Control, Fusarium oxysporum f. sp. \\ cubense, Musa sp., Plantains, Pseudomonas aeruginosa
}

\section{Introduction}

The interactions between endophytic microorganisms and host plants and the relevance of such integrally associated microbes in crop production and protection form emerging areas of research in plant sciences [1] [2]. Endophytic bacteria have major roles in plant growth including growth promotion [3], biocontrol of pathogens [4] and pests [5]. Earlier cultivation-based studies have indicated endophytic bacteria, as predominantly root colonizers often present in fewer numbers [6]. Application of cultivation independent molecular techniques has brought out the prevalence of considerable amount of bacterial endophytes in different plant species, mostly in a noncultivable form [2] [7]. Cultivation-based previous studies in banana cv. Grand Naine revealed the presence of a wide range of endophytes both in field shoot-tip region and in micropropagated in vitro stocks [8] [9]. Recent studies employing different banana cultivars have revealed the extensive inhabitation of shoot tissues including the intracellular niches by normally non-cultivable bacterial endophytes. This included colonization in the cytoplasm and in the cell wall-plasma membrane peri-space with the designation of the organisms in the above two niches as "Cytobacts" and "Peribacts" respectively [10] [11]. Although a vast share of endophytes exist in a non-cultivable form, cultivation-based studies and community analysis are warranted for further exploitation of organisms in crop production and protection.

Banana wilt caused by the fungal pathogen Fusarium oxysporum Schlect f. sp. cubense (E. F. Smith) Snyd. \& Hans. (Foc), widely referred to as Panama disease, is a devastating disease of bananas and plantains (Musa sp.) throughout the world [12]-[14]. The first description of Fusarium wilt in banana and plantains came from Australia [13] [15]. The disease is now recognized as the most destructive one limiting banana cultivation all over the world [13] [14] [16]. The pathogen is soil-borne invading the roots and obstructing the water and nutrient flow through vascular colonization. Consequently, leaves turn yellow with the oldest ones succumbing first. The plants eventually wilt and collapse, thus causing serious crop losses [13] [15]. The pathogen is broadly categorised into four races, of which races 1, 2 and 4 are pathogenic to banana, with race 1 infecting most banana cultivars excluding "Cavendish group" [13] [14] [17]. In India, the disease is prevalent in all banana growing areas with race 1 forming the most common one followed by race 2 [16] [18], while race 4 has been reported in certain pockets [19].

Conventional measures to control the pathogen include crop rotation, stringent adoption of phytosanitary measures and the use of resistant varieties [13] [14] [16]. Chemical control measures are often impractical and ineffective. Biological control of plant pathogens employing antagonistic microorganisms is considered as an environment-friendly and cost effective approach. Fusarium wilt in banana is reported to be controlled by Pseudomonas fluorescence [18], Bacillus subtilis [20] and the endophytic Burkholderia cenocepacia [21]. Endophytes colonizing the vascular system which constitutes the same niche as Foc form potential candidates for developing biocontrol strategies. This study was undertaken with the aim of isolating the bacterial endophytes associated with field-derived banana suckers and evaluating them for the antagonistic activity against Foc.

\section{Materials and Methods}

\subsection{Nutrient Media}

Two commonly employed bacteriological media, namely nutrient agar (NA: bacteriological peptone $5 \mathrm{~g} \cdot \mathrm{l}^{-1}, \mathrm{NaCl} 5$ $\mathrm{g} \cdot \mathrm{l}^{-1}$, beef extract $3 \mathrm{~g} \cdot \mathrm{l}^{-1}$, agar $15 \mathrm{~g} \cdot \mathrm{l}^{-1}, \mathrm{pH} 6.8 \pm 0.2$ ) and trypticase soy agar (TSA: peptone from casein $17 \mathrm{~g} \cdot \mathrm{l}^{-1}$, 
peptone from soymeal $3 \mathrm{~g} \cdot \mathrm{l}^{-1}$, dextrose $2.5 \mathrm{~g} \cdot \mathrm{l}^{-1}, \mathrm{NaCl} 5 \mathrm{~g} \cdot \mathrm{l}^{-1}, \mathrm{~K}_{2} \mathrm{HPO}_{4} 2.5 \mathrm{~g} \cdot \mathrm{l}^{-1}$, agar $15 \mathrm{~g} \cdot \mathrm{l}^{-1}$, pH 7.2 \pm 0.2$)$ were used for the bacterial isolation and further characterization. The nutrient plates were monitored prior to use for $4-7$ days to ensure freedom from all microbial contaminants [7] [8]. Potato dextrose agar (PDA: potato infusion from $200 \mathrm{~g} \cdot \mathrm{l}^{-1}$, dextrose $20 \mathrm{~g} \cdot \mathrm{l}^{-1}$, agar $15 \mathrm{~g} \cdot \mathrm{l}^{-1}$ ) and NA were employed for fungal-bacterial dual culture experiments while PDA was used for normal fungal culturing.

\subsection{Surface Sterilization and Preparation of Plant Material}

Suckers of banana cv. Grand Naine (2 - 3 months old) were collected from the institute (IIHR) farm. The suckers were disinfected as per as per Thomas et al. [7]. Briefly, the rhizome and pseudostem parts were chopped down and the central $5-6 \mathrm{~cm}$ tissue was incubated in $0.5 \%$ Bavistin ${ }^{\circledR}$ (Carbendazim 50\% WP) overnight at 150 $\mathrm{rpm}$ in a rotary shaker. After a rinse in sterile water, the sucker was trimmed down to $3-4 \mathrm{~cm}$ and treated with $0.05 \%$ cetrimide for $1 \mathrm{~h}$. All subsequent steps were performed in a laminar air flow (LAF) cabinet fitted with an Ultra-Low Penetration Air filter (Esco Biotechnology Equipment Division, Singapore). After removing the external leaf sheaths, the shoot-tip part was treated with $\mathrm{NaOCl}$ (4\% available chlorine) for 20 min followed by 8 rinses in sterile water. The last two wash solutions were plated on NA and TSA to confirm the effectiveness of disinfection treatments. Shoot-tips of $1-1.5 \mathrm{~cm}$ were collected after removing the external oxidized tissue and were used for bacterial isolation soon after.

\subsection{Tissue Homogenization and Plating}

Ten suckers were used for the bacterial isolation during August 2013 to February 2014 in five batches of two suckers each. The excised $1-1.5 \mathrm{~cm}$ shoot-tips comprising of half pseudostem and corm tissue were cut longitudinally one half of which was used for bacterial isolation. The tissue was ground in a mortar employing sterile peptone salt $\left(1 \mathrm{~g} \cdot \mathrm{l}^{-1}\right.$ each of peptone and $\left.\mathrm{NaCl}\right)$ [22]. The homogenate was allowed to stand for $20-30 \mathrm{~min}$ at $4^{\circ} \mathrm{C}$ and the supernatant $\left(10^{\circ}\right)$ and three decimal serial dilutions $\left(10^{1}\right.$ and $\left.10^{3}\right)$ were spread on NA and TSA through the spotting and tilt spreading (SATS) method [22] employing three replications per dilution. The NA pates were incubated at $37^{\circ} \mathrm{C}$ and TSA plates at $30^{\circ} \mathrm{C}$ for a week and observed for the bacterial colony growth. Thereafter the plates were monitored at room temperature up to one month.

\subsection{Identification of Bacterial Isolates}

Distinct colony types were picked up from NA and TSA plates for each sucker and were purified through three rounds of streaking and single colony selection on the same medium. The purified cultures were grown together on either media by single colony spotting to identify the distinct colony types, thus to avoid duplications. The identity of the organisms was established through 16S rRNA gene sequence based homology analysis. For this, bacterial DNA was isolated employing the AxyPrep Bacterial Genomic DNA Miniprep kit (Axygen Biosciences, USA) and 16S rRNA gene amplification was performed through PCR using universal bacterial primers $27 \mathrm{~F}$ (5'-AGAGTTTGATCCTGGCTCAG-3') and 1492R-Y (5'-GGYTACCTTGTTACGACTT-3'; Y = C/T) as described elsewhere [7]. The thermocycling conditions included initial one denaturation step of $94^{\circ} \mathrm{C}$ for 5 min followed by 35 amplification cycles of $94^{\circ} \mathrm{C}$ for $30 \mathrm{~s}, 55^{\circ} \mathrm{C}$ for $40 \mathrm{~s}, 72^{\circ} \mathrm{C}$ for $40 \mathrm{~s}$ followed by a final extension at $72^{\circ} \mathrm{C}$ for $5 \mathrm{~min}$ [7] [9]. After confirming PCR amplification in a $1 \%$ agarose gel and column purification of the PCR product (Macherey-Nagel GmbH, Germany), the 16SrRNA gene was single end-sequenced (Xcelris Genomics, Ahmedabad, India) using $27 \mathrm{~F}$ primer. The identity of the organisms was determined by megablast analysis at the NCBI Gen Bank database (http://blast.ncbi.nlm.nih.gov/Blast.cgi), and was validated through Seqmatch search at the Ribosomal Database Project (http://rdp.cme.msu.edu/seqmatch/seqmatch_intro.jsp).

\subsection{Confirming the Identity of Fungal Pathogen}

The culture of pathogen (Foc, race 1), supplied by Dr. T. R. Usharani of this institute (IIHR), was purified through three rounds of hyphal tip culturing in PDA at room temperature $\left(26^{\circ} \mathrm{C}-28^{\circ} \mathrm{C}\right)$. Mycelia from PDA culture were used for DNA isolation employing MOBIO microbial DNA isolation kit. The ITS region was amplified in PCR with the primers ITS1 (5'-TCCGTAGGTGAACCTGCGG-3') and ITS4 (5'-TCCTCCGCTTATTGATATGC-3') [23] employing the thermocycling conditions: $95^{\circ} \mathrm{C}$ for $5 \mathrm{~min}$ followed by 35 cycles of $94^{\circ} \mathrm{C}$ for $30 \mathrm{sec}, 53^{\circ} \mathrm{C}$ for $40 \mathrm{sec}, 72^{\circ} \mathrm{C}$ for $30 \mathrm{sec}$ and a final extension of $72^{\circ} \mathrm{C}$ for $10 \mathrm{~min}$ as per our optimization. PCR products were 
column purified as above and sequenced with the ITS1 primer. The identity was established through megablast analysis at the NCBI GenBank as detailed earlier.

\subsection{Fungal Antagonistic Assays}

The endophytic isolates were tested for the potential antagonistic activity against the pathogen through agar plate assay. In the initial trial, 0.1 OD suspension of overnight culture of $F o c$ in potato dextrose broth (PDB) was plated onto PDA and NA in $10 \mathrm{~cm}$ diameter plates (Hi Media Biosciences, Mumbai). After surface drying, pits of $5-6 \mathrm{~mm}$ diameter were prepared with the distal end of $200 \mu \mathrm{l}$ sterile pipette tips and $50 \mu \mathrm{l}$ of $1.0 \mathrm{OD}$ suspensions of different bacterial isolates were applied singly followed by surface-drying in the LAF. The plates were incubated at room temperature and observed for fungal lawn formation and inhibition zone development for 2 - 7 days. The experiment was repeated twice. As an alternate approach, adding the test bacterial suspensions as $10 \mu \mathrm{l}$ spots on the previously Foc-spread PDA and NA plates was tried.

A pathogen-antagonist confrontation assay was performed with the selected bacterial isolates. For this, PDA, NA and NA + PDA (1:1) plates were spotted at the edges with 1.0 OD suspension of four test isolates per plate and an agar plug (5 mm diameter) cut from an actively growing PDA culture of $F o c$ was placed at the centre. Control plates contained the mycelial plug placed similarly at the centre without bacterial cultures. The plates were observed for up to 2 weeks for the extent of fungal growth.

\subsection{Accession Numbers}

The 16SrRNA gene sequence data of the endophytic bacterial isolates identified in this study have been deposited with the NCBI GenBank with the acc. nos. KP798811 to KP798857 (Table 1).

\section{Results}

\subsection{Endophytic Bacterial Population in Banana Shoot-Tips}

The ten suckers showed considerable variation in the population of cultivable bacteria (CFU in the range of 3.0 $\times 10^{1}$ to $5.1 \times 10^{4} \mathrm{~g}^{-1}$ tissue fresh weight) with the number of distinct isolates per sucker varying from one to 15 (Figure 1). Altogether, 47 isolates were obtained which included 26 from NA and 21 from TSA. Once revived, all of the isolates showed growth on either media. NA was employed as the choice medium during further bacterial culturing works. The wash solutions upon plating did not yield any bacterial growth confirming the effectiveness of surface sterilization treatments.

\subsection{Bacterial Identification}

The PCR products were single-end sequenced yielding $>500$ bp 5'-16S rRNA nucleotide data in most instances. The identity of the isolates was established through megablast analysis at the NCBI database which was further

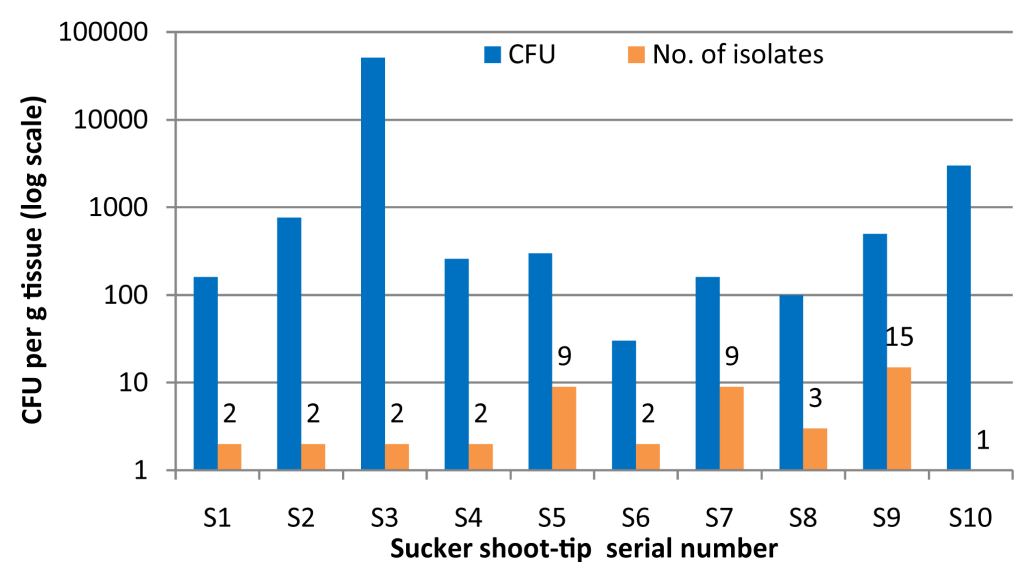

Figure 1. Endophytic bacterial $\mathrm{CFU} \cdot \mathrm{g}^{-1}$ shoot-tip tissue in individual suckers (S) of banana cv. Grand Naine and the number of bacterial isolates per sucker. 
Table 1. Endophytic bacteria isolated from banana cv. Grand Naine identified based on partial 16S rRNA sequence homology analysis through megablast at NCBI GenBank and Seqmatch at RDP database.

\begin{tabular}{|c|c|c|c|c|c|c|}
\hline S. No. & Isolate ID & $\begin{array}{l}\text { 16SrRNA } \\
\text { seq. (bp) }\end{array}$ & $\begin{array}{l}\text { NCBI } \\
\text { acc. no. }\end{array}$ & $\begin{array}{l}\text { Closest species match } \\
\text { in NCBI GenBank with } \\
\text { acc. no. and homology }\end{array}$ & $\begin{array}{l}\text { Closest species match } \\
\text { in RDP with acc. no. } \\
\text { and similarity score }\end{array}$ & $\begin{array}{l}\text { Suggested } \\
\text { identification } \\
\text { and phylogeny }\end{array}$ \\
\hline 1 & GNS13.1a & 615 & KP798811 & $\begin{array}{l}\text { Micrococcus endophyticus } \\
\text { (AB931118;99\%) }\end{array}$ & $\begin{array}{l}\text { Micrococcus endophyticus } \\
\text { (S000893772, 0.977) }\end{array}$ & $\begin{array}{l}\text { M. endophyticus; } \\
\text { Actinobacteria }\end{array}$ \\
\hline 2 & GNS13.1b & 754 & KP798812 & $\begin{array}{l}\text { Bacillus safensis } \\
\text { (LC015558; 100\%) }\end{array}$ & $\begin{array}{c}\text { Bacillus safensis } \\
(\mathrm{S} 000458519,1.000)\end{array}$ & $\begin{array}{l}\text { B. safensis; } \\
\text { Firmicutes }\end{array}$ \\
\hline 3 & GNS13.2a & 760 & KP798813 & $\begin{array}{l}\text { Pseudomonas aeruginosa } \\
\quad(\mathrm{CP} 010555 ; 100 \%)\end{array}$ & $\begin{array}{l}\text { Pseudomonas aeruginosa } \\
\quad(\mathrm{S} 003288366,1.000)\end{array}$ & $\begin{array}{l}\text { P. aeruginosa; } \\
\gamma \text { Proteobacteria }\end{array}$ \\
\hline 4 & GNS13.2b & 633 & KР798814 & $\begin{array}{l}\text { Kytococcus sedentarius } \\
\text { (EU443746; 99\%) }\end{array}$ & $\begin{array}{l}\text { Kytococcus sedentarius } \\
\text { (S000007341, 0.974) }\end{array}$ & $\begin{array}{l}\text { K. sedentarius; } \\
\text { Actinobacteria }\end{array}$ \\
\hline 5 & GNS13.3a & 751 & KP798815 & $\begin{array}{l}\text { Klebsiella variicola } \\
(\mathrm{KC} 853303 ; 100 \%)\end{array}$ & $\begin{array}{l}\text { Klebsiella variicola } \\
\text { (S000324392, 0.990) }\end{array}$ & $\begin{array}{c}\text { K. variicola; } \\
\gamma \text { Proteobacteria }\end{array}$ \\
\hline 6 & GNS13.3b & 760 & KP798816 & $\begin{array}{l}\text { Pseudomonas putida } \\
\text { (HQ236534; 100\%) }\end{array}$ & $\begin{array}{l}\text { Pseudomonas taiwanensis } \\
\quad(\mathrm{S} 001095516,0.999)\end{array}$ & $\begin{array}{c}\text { P. putida; } \\
\gamma \text { Proteobacteria }\end{array}$ \\
\hline 7 & GNS13.4a & 751 & KP798817 & $\begin{array}{l}\text { Enterobacter cloacae } \\
(\mathrm{KM} 077045 ; 100 \%)\end{array}$ & $\begin{array}{l}\text { Enterobacter cloacae } \\
(\mathrm{S} 000021184,0.990)\end{array}$ & $\begin{array}{c}\text { E. cloacae; } \\
\gamma \text { Proteobacteria }\end{array}$ \\
\hline 8 & GNS13.4b & 771 & KP798818 & $\begin{array}{l}\text { Staphylococcus pasteuri } \\
\text { (KJ767309; 100\%) }\end{array}$ & $\begin{array}{l}\text { Staphylococcus pasteuri } \\
\text { (S000381991, 1.000) }\end{array}$ & $\begin{array}{l}\text { S. pasteuri; } \\
\text { Firmicutes }\end{array}$ \\
\hline 9 & GNS13.5a & 876 & KР798819 & $\begin{array}{l}\text { Sphingomonas mucosissima } \\
\text { (KF924237; 100\%) }\end{array}$ & $\begin{array}{l}\text { Sphingomonas aurantiaca } \\
(\mathrm{S} 000145110,0.946)\end{array}$ & $\begin{array}{l}\text { S. mucosissima; } \\
\alpha \text { Proteobacteria }\end{array}$ \\
\hline 10 & GNS13.5b & 423 & KP798820 & $\begin{array}{l}\text { Micrococcus luteus } \\
(\mathrm{KF} 891348 ; 100 \%)\end{array}$ & $\begin{array}{l}\text { Micrococcus luteus } \\
\text { (S000110436, 0.857) }\end{array}$ & $\begin{array}{l}\text { M. luteus; } \\
\text { Actinobacteria }\end{array}$ \\
\hline 11 & GNS13.5c & 885 & KP798821 & $\begin{array}{l}\text { Brevundimonas vesicularis } \\
\text { (KC494336; 99\%) }\end{array}$ & $\begin{array}{l}\text { Brevundimonas nasdae } \\
\text { (S000334613, 0.954) } \\
\text { Brevundimonas vesicularis } \\
\text { (S000112396, 0.952) }\end{array}$ & $\begin{array}{l}\text { B. vesicularis; } \\
\alpha \text { Proteobacteria }\end{array}$ \\
\hline 12 & GNS13.5d & 451 & KP798822 & $\begin{array}{l}\text { Kocuria palustris } \\
\text { (KF424691; 98\%) }\end{array}$ & $\begin{array}{c}\text { Kocuria palustris } \\
(\mathrm{S} 000014978,0.875)\end{array}$ & $\begin{array}{l}\text { K. palustris; } \\
\text { Actinobacteria }\end{array}$ \\
\hline 13 & GNS13.5e & 681 & KP798823 & $\begin{array}{l}\text { Sphingomonas panni } \\
(\mathrm{HQ} 739092 ; 99 \%)\end{array}$ & $\begin{array}{l}\text { Sphingomonas panni } \\
(\mathrm{S} 000471092,0.949)\end{array}$ & $\begin{array}{c}\text { S. panni; } \\
\alpha \text { Proteobacteria }\end{array}$ \\
\hline 14 & GNS13.5f & 601 & KP798824 & $\begin{array}{l}\text { Micrococcus yunnanensis } \\
\quad(\mathrm{KF} 424616 ; 98 \%)\end{array}$ & $\begin{array}{l}\text { Micrococcus yunnanensis } \\
\text { (S001241135, 0.857) }\end{array}$ & $\begin{array}{l}\text { M. yunnanensis; } \\
\text { Actinobacteria }\end{array}$ \\
\hline 15 & GNS13.5g & 611 & KP798825 & $\begin{array}{l}\text { Micrococcus luteus } \\
\text { (KF358260; 98\%) }\end{array}$ & $\begin{array}{l}\text { Micrococcus endophyticus } \\
\text { (S000893772, 0.861) }\end{array}$ & $\begin{array}{l}\text { Micrococcus sp.; } \\
\text { Actinobacteria }\end{array}$ \\
\hline 16 & GNS13.5h & 691 & KP798826 & $\begin{array}{c}\text { Staphylococcus epidermidis } \\
\text { (KP053611;99\%) }\end{array}$ & $\begin{array}{l}\text { Staphylococcus epidermidis } \\
\text { (S000413964, 0.993) }\end{array}$ & $\begin{array}{l}\text { S. epidermidis; } \\
\text { Firmicutes }\end{array}$ \\
\hline 17 & GNS13.5i & 641 & KP798827 & $\begin{array}{l}\text { Sphingomonas mucosissima } \\
\text { (KM272391; 100\%) }\end{array}$ & $\begin{array}{l}\text { Sphingomonas aurantiaca } \\
(\mathrm{S} 000145110,0.949)\end{array}$ & $\begin{array}{l}\text { S. mucosissima; } \\
\alpha \text { Proteobacteria }\end{array}$ \\
\hline 18 & GNS13.6a & 618 & KP798828 & $\begin{array}{c}\text { Bacillus safensis } \\
\text { (LC015558; 100\%) }\end{array}$ & $\begin{array}{c}\text { Bacillus safensis } \\
(\mathrm{S} 000458519,1.000)\end{array}$ & $\begin{array}{l}\text { B. safensis; } \\
\text { Firmicutes }\end{array}$ \\
\hline 19 & GNS13.6b & 561 & KР798829 & $\begin{array}{l}\text { Serratia marcescens } \\
(\mathrm{KF} 624754 ; 100 \%)\end{array}$ & $\begin{array}{l}\text { Serratia nematodiphila } \\
(\mathrm{S} 000903064,1.000)\end{array}$ & $\begin{array}{l}\text { S. marcescens; } \\
\gamma \text { Proteobacteria }\end{array}$ \\
\hline 20 & GNS13.7a & 556 & KP798830 & $\begin{array}{c}\text { Arthrobacter chlorophenolicus } \\
\text { (JQ958834; 100\%) } \\
\text { Arthrobacter globiformis } \\
\text { (FN178398; 100\%) }\end{array}$ & $\begin{array}{c}\text { Arthrobacter equi }(\mathrm{S} 002033220, \\
0.981)\end{array}$ & $\begin{array}{l}\text { Arthrobacter sp.; } \\
\text { Actinobacteria }\end{array}$ \\
\hline 21 & GNS13.7b & 666 & KP798831 & $\begin{array}{c}\text { Curtobacterium } \\
\text { oceanosedimentum } \\
\text { (KM019700; 99\%) }\end{array}$ & $\begin{array}{l}\text { Curtobacterium pusillum } \\
\text { (S000367091, 0.981) }\end{array}$ & $\begin{array}{l}\text { C. oceanosedimentum; } \\
\text { Actinobacteria }\end{array}$ \\
\hline 22 & GNS13.7c & 529 & KP798832 & $\begin{array}{c}\text { Brevibacterium pityocampae } \\
\text { (JQ388726;97\%) }\end{array}$ & $\begin{array}{l}\text { Brevibacterium pityocampae } \\
\quad(\mathrm{S} 001016069,0.753)\end{array}$ & $\begin{array}{l}\text { Brevibacterium sp.; } \\
\text { Actinobacteria }\end{array}$ \\
\hline
\end{tabular}




\section{Continued}

\begin{tabular}{|c|c|c|c|c|c|c|}
\hline 23 & GNS13.7d & 521 & KP798833 & $\begin{array}{l}\text { Corynebacterium } \\
\text { lipophiloflavum } \\
\text { (KF777371; 99\%) }\end{array}$ & $\begin{array}{c}\text { Corynebacterium } \\
\text { lipophiloflavum } \\
(\mathrm{S} 000011673,0.901)\end{array}$ & $\begin{array}{l}\text { C. lipophiloflavum; } \\
\text { Actinobacteria }\end{array}$ \\
\hline 24 & GNS13.7e & 600 & KP798834 & $\begin{array}{l}\text { Kytococcus sedentarius } \\
\quad(\text { EU443746; 99\%) }\end{array}$ & $\begin{array}{l}\text { Kytococcus sedentarius } \\
\text { (S000007341, 0.967) }\end{array}$ & $\begin{array}{l}\text { K. sedentarius; } \\
\text { Actinobacteria }\end{array}$ \\
\hline 25 & GNS13.7g & 503 & KP798835 & $\begin{array}{l}\text { Serratia nematodiphila } \\
\quad(\mathrm{KJ} 535365 ; 99 \%)\end{array}$ & $\begin{array}{l}\text { Serratia nematodiphila } \\
\text { (S000903064 0.949) }\end{array}$ & $\begin{array}{l}\text { S. nematodiphila } \\
\gamma \text { Proteobacteria }\end{array}$ \\
\hline 26 & GNS13.7h & 641 & KP798836 & $\begin{array}{c}\text { Serratia marcescens } \\
(\mathrm{KF} 624754 ; 100 \%)\end{array}$ & $\begin{array}{l}\text { Serratia marcescens } \\
(\mathrm{S} 0001164230.990)\end{array}$ & $\begin{array}{l}\text { S. marcescens; } \\
\gamma \text { Proteobacteria }\end{array}$ \\
\hline 27 & GNS13.7j & 616 & KP798837 & $\begin{array}{c}\text { Brevibacterium } \\
\text { pityocampae (NR116332; 98\%) }\end{array}$ & $\begin{array}{l}\text { Brevibacterium pityocampae } \\
\quad(\mathrm{S} 001016069,0.848)\end{array}$ & $\begin{array}{c}\text { Brevibacterium sp.; } \\
\text { Actinobacteria }\end{array}$ \\
\hline 28 & GNS13.7k & 610 & KP798838 & $\begin{array}{c}\text { Brevibacterium pityocampae } \\
\text { (NR116332; 98\%) }\end{array}$ & $\begin{array}{l}\text { Brevibacterium pityocampae } \\
\quad(\mathrm{S} 001016069,0.837)\end{array}$ & $\begin{array}{c}\text { Brevibacterium sp.; } \\
\text { Actinobacteria }\end{array}$ \\
\hline 29 & GNS13.8a & 661 & KP798839 & $\begin{array}{l}\text { Kocuria rhizophila } \\
(\mathrm{KC} 429605 ; 99 \%)\end{array}$ & $\begin{array}{l}\text { Kocuria rhizophila } \\
(\mathrm{S} 000008149,0.978)\end{array}$ & $\begin{array}{l}\text { K. rhizophila; } \\
\text { Actinobacteria }\end{array}$ \\
\hline 30 & GNS13.8b & 599 & KP798840 & $\begin{array}{c}\text { Jatrophihabitans endophyticus } \\
\text { (NR109586; 95\%); } \\
\text { Streptomyces aomiensis } \\
\text { (NR112998; 95\%); } \\
\text { Streptomyces catbensis; } \\
\text { (NR125457; 93\%) }\end{array}$ & $\begin{array}{c}\text { Actinokineospora } \\
\text { cibodasensis } \\
(\mathrm{S} 001151982,0.708)\end{array}$ & $\begin{array}{l}\text { Actinobacteria; } \\
\text { Frankiaceae }\end{array}$ \\
\hline 31 & GNS13.8c & 606 & KP798841 & $\begin{array}{l}\text { Tessaracoccus flavescens } \\
\text { (JQ897419;96\%) }\end{array}$ & $\begin{array}{l}\text { Tessaracoccus lubricantis } \\
\quad(\mathrm{S} 001156636,0.758)\end{array}$ & $\begin{array}{c}\text { Tessaracoccus sp.; } \\
\text { Actinobacteria }\end{array}$ \\
\hline 32 & GNS13.9a & 660 & KP798842 & $\begin{array}{l}\text { Klebsiella pneumoniae } \\
\quad(\mathrm{JF} 489150 ; 100 \%)\end{array}$ & $\begin{array}{l}\text { Klebsiella pneumoniae } \\
\text { (S000021704, 0.991) }\end{array}$ & $\begin{array}{l}\text { K. pneumoniae; } \\
\gamma \text { Proteobacteria }\end{array}$ \\
\hline 33 & GNS13.9b & 661 & KР798843 & $\begin{array}{c}\text { Rothia terrae } \\
\text { (KM577655; 99\%) }\end{array}$ & $\begin{array}{c}\text { Rothia terrae } \\
(\mathrm{S} 000722242,0.972)\end{array}$ & $\begin{array}{c}\text { R. terrae; } \\
\text { Actinobacteria }\end{array}$ \\
\hline 34 & GNS13.9c & 546 & KР798844 & $\begin{array}{c}\text { Rothia terrae } \\
\text { (KM577655; 99\%) }\end{array}$ & $\begin{array}{c}\text { Rothia terrae } \\
(\mathrm{S} 000722242,0.964)\end{array}$ & $\begin{array}{c}\text { R. terrae; } \\
\text { Actinobacteria }\end{array}$ \\
\hline 35 & GNS13.9d & 656 & KP798845 & $\begin{array}{c}\text { Rothia amarae } \\
\text { (FR682692; 100\%) }\end{array}$ & $\begin{array}{c}\text { Rothia amarae } \\
(\mathrm{S} 000484536,1.000)\end{array}$ & $\begin{array}{c}\text { R. terrae; } \\
\text { Actinobacteria }\end{array}$ \\
\hline 36 & GNS13.9e & 712 & KP798846 & $\begin{array}{l}\text { Staphylococcus pasteuri } \\
\text { (KJ767309; 100\%) }\end{array}$ & $\begin{array}{l}\text { Staphylococcus pasteuri } \\
(\mathrm{S} 000381991,1.000)\end{array}$ & $\begin{array}{l}\text { S. pasteuri; } \\
\text { Firmicutes }\end{array}$ \\
\hline 37 & GNS13.9f & 668 & KP798847 & $\begin{array}{l}\text { Staphylococcus pasteuri } \\
\text { (KP261076; 99\%) }\end{array}$ & $\begin{array}{l}\text { Staphylococcus pasteuri } \\
\text { (S000381991, 0.992) }\end{array}$ & $\begin{array}{l}\text { S. pasteuri; } \\
\text { Firmicutes }\end{array}$ \\
\hline 38 & GNS13.9g & 712 & KP798848 & $\begin{array}{c}\text { Rothia amarae } \\
\text { (FR682692; 100\%) }\end{array}$ & $\begin{array}{c}\text { Rothia amarae } \\
\text { (S000484536 1.000) }\end{array}$ & $\begin{array}{c}\text { R. amarae; } \\
\text { Actinobacteria }\end{array}$ \\
\hline 39 & GNS13.9h & 650 & KР798849 & $\begin{array}{l}\text { Staphyloccoccus warneri } \\
\text { (KP261060; 99\%) }\end{array}$ & $\begin{array}{c}\text { Staphylococcus warneri } \\
\text { (S000414717, 0.986) }\end{array}$ & $\begin{array}{l}\text { S. warneri; } \\
\text { Firmicutes }\end{array}$ \\
\hline 40 & GNS13.9i & 604 & KP798850 & $\begin{array}{c}\text { Rothia terrae } \\
\text { (KM577655; 100\%) }\end{array}$ & $\begin{array}{c}\text { Rothia terrae } \\
(\mathrm{S} 000722242,0.990)\end{array}$ & $\begin{array}{c}\text { R. terrae; } \\
\text { Actinobacteria }\end{array}$ \\
\hline 41 & GNS13.9j & 519 & KP798851 & $\begin{array}{c}\text { Rothia terrae } \\
\text { (HQ908710; 99\%) }\end{array}$ & $\begin{array}{c}\text { Rothia terrae } \\
(\mathrm{S} 000722242,0.927)\end{array}$ & $\begin{array}{c}\text { R. terrae; } \\
\text { Actinobacteria }\end{array}$ \\
\hline 42 & GNS13.9k & 686 & KP798852 & $\begin{array}{c}\text { Rothia amarae } \\
\text { (FR682692; 100\%) }\end{array}$ & $\begin{array}{c}\text { Rothia amarae } \\
(\mathrm{S} 000484536,1.000)\end{array}$ & $\begin{array}{c}\text { R. terrae; } \\
\text { Actinobacteria }\end{array}$ \\
\hline 43 & GNS13.91 & 693 & KP798853 & $\begin{array}{l}\text { Bacillus okhensis } \\
\text { (NR043484; 99\%) }\end{array}$ & $\begin{array}{c}\text { Bacillus okhensis } \\
(\mathrm{S} 000653122,0.994)\end{array}$ & $\begin{array}{l}\text { B. okhensis; } \\
\text { Firmicutes }\end{array}$ \\
\hline 44 & GNS13.9n & 696 & KР798854 & $\begin{array}{l}\text { Naumannella halotolerans } \\
\quad(\text { FR832426; 100\%) }\end{array}$ & $\begin{array}{c}\text { Auraticoccus monumenti } \\
(\mathrm{S} 002032927,0.773)\end{array}$ & $\begin{array}{l}\text { N. halotolerans; } \\
\text { Actinobacteria }\end{array}$ \\
\hline 45 & GNS13.9o & 691 & KP798855 & $\begin{array}{c}\text { Micrococcus terreus } \\
\text { (HQ908754; 100\%) }\end{array}$ & $\begin{array}{l}\text { Micrococcus terreus } \\
(\mathrm{S} 001241593,0.967)\end{array}$ & $\begin{array}{c}\text { M. terreus; } \\
\text { Actinobacteria }\end{array}$ \\
\hline 46 & GNS13.9p & 601 & KP798856 & $\begin{array}{l}\text { Kytococcus sedentarius } \\
\text { (KJ732936; 99\%) }\end{array}$ & $\begin{array}{l}\text { Kytococcus sedentarius } \\
\text { (S000007341, 0.974) }\end{array}$ & $\begin{array}{l}\text { K. sedentarius; } \\
\text { Actinobacteria }\end{array}$ \\
\hline 47 & GNS13.10a & 639 & KP798857 & $\begin{array}{l}\text { Staphylococcus hominis } \\
\text { (KM603639; 100\%) }\end{array}$ & $\begin{array}{l}\text { Staphylococcus hominis } \\
\text { (S000016099 0.989) }\end{array}$ & $\begin{array}{l}\text { S. hominis; } \\
\text { Firmicutes }\end{array}$ \\
\hline
\end{tabular}


validated through Seqmatch at RDP (Table 1). Wherever any differences in the species identity appeared between the two databases, the identity was fixed based on the more number of homologous matches at NCBI or higher scores towards a particular species at RDP. The identity was established to the species level in $85 \%$ instances and to genus level in 13\% cases, but not for one isolate, GNS.13.8b, which showed the maximum homology score of $95 \%$ to Jatrophihabitans endophyticus and Streptomyces aomiensis (95\%) in the NCBI database followed by Streptomyces catbensis (93\%). The maximum homology in RDP database was to Actinokineospora cibodasensis (score 0.708 ). Therefore, this isolate could be assigned only up to the family level, namely, Frankiaceae. The isolate GNS.13.8c also displayed a low homology to the closest match in NCBI (Tessaracoccus flavescens; 96\%) and low RDP score (Tessaracoccus lubricantis; 0.758 ) but could be identified to the genus level. Thus, the two isolates GNS.13.8b and GNS.13.8c appeared to be novel organisms which demands additional inputs for their proper characterization.

The 47 endophytic isolates belonged to the phylogenetic classes of Actinobacteria, $\alpha$ - and $\gamma$-Proteobacteria and spore-forming and non-spore-forming Firmicutes (Figure 2). Actinobacteria constituted the predominant class, also displaying the highest diversity. These included Arthrobacter, Brevibacterium, Corynebacterium, Curtobacterium, Kocuria, Kytococcus, Micrococcus, Naumanella, Rothia, Tessaracoccus spp. and the unidentified GNS.13.8b isolate (Figure 3). Proteobacteria group included Sphingomonas mucosissimas, S. panni and Brevundimonas vesicularis from $\alpha$-subclass, and Pseudomonas aeruginosa, P. putida, Klebsiella variicola, $K$. pneumonia, Enterobacter cloacae, Serratia marcescens and S. nematodiphila from $\gamma$-subclass. Spore-forming Firmicutes included Bacillus safensis and the uncommon B. okhensis. Non-sporulating Firmicutes included Staphylococcus epidermidis, S. pasteuri, S. hominis and S. warneri. Thus, several uncommon organisms were obtained as endophytic bacterial associates from "Grand Naine" shoot-tips.

\subsection{Identification of Fungal Pathogen}

PCR with ITS1 and ITS4 primers gave an amplicon of about $500 \mathrm{bp}$. Sequencing of the purified PCR product with primer ITS1 yielded 511 bp data. The megablast analysis at the NCBI GenBank showed $100 \%$ homology to Fusarium oxysporum f. sp. cubense.

\subsection{Fungal Antagonistic Assay}

A uniform lawn of $F o c$ was obtained on PDA and NA within 4 - 7 days of incubation at room temperature. Among the 47 bacterial isolates tested, only one organism, namely Pseudomonas aeruginosa (GNS.13.2a) showed significant antagonistic activity against the pathogen in pit method of screening on both NA and PDA (Figure 4). Two other organisms, namely Klebsiella variicola (GNS13.3a) and Enterobacter cloacae (GNS13.4a) showed relatively mild zone of inhibition. Similar results were obtained in the spot method of application (Figure 5). In confrontation assay, which was undertaken on NA+PDA medium, $P$. aeruginosa showed clear inhibition of fungal growth while the effect was less prominent for the other two (Figure 6).

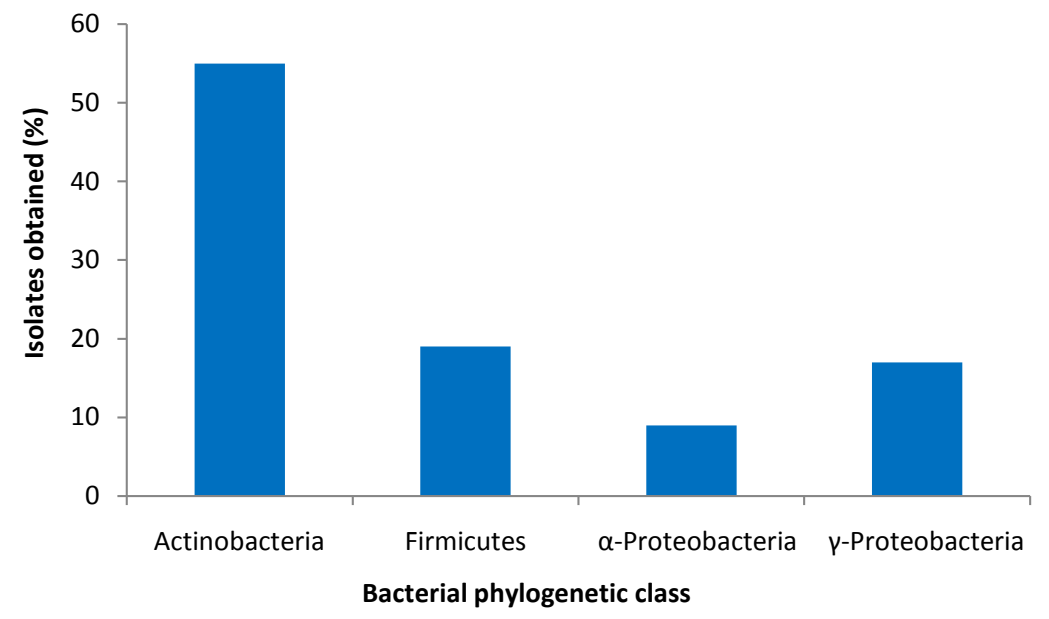

Figure 2. Frequency distribution of endophytic bacterial isolates from shoot-tip tissue of banana based phylogenetic groups. 


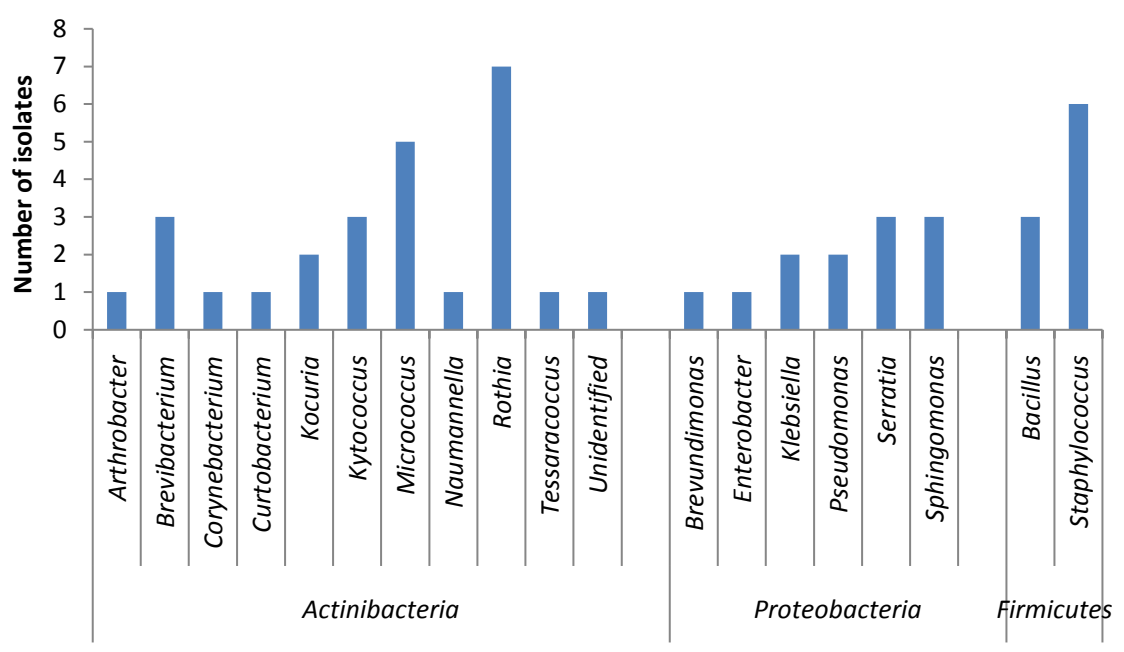

Bacterial class/genera

Figure 3. Diversity of endophytic bacteria isolated from shoot-tip tissue of banana cv. Grand Naine.

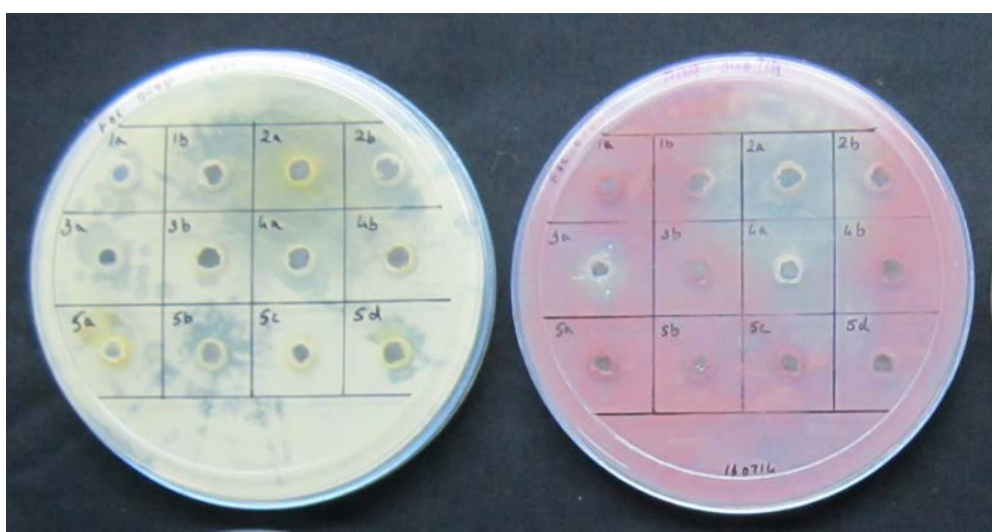

Figure 4. Screening of banana bacterial endophytes for antagonistic activity against Fusarium oxysporum $\mathrm{f}$. sp. cubense on NA (left) and PDA (right) through pit-inoculation approach: 2a, 3a and 4a showing inhibition zone correspond to Pseudomonas aeruginosa, Klebsiella variicola and Enterobacter cloacae, respectively.

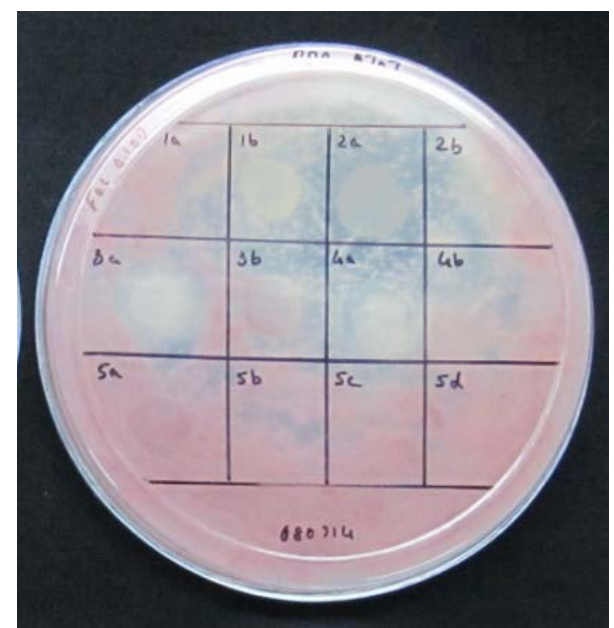

Figure 5. Screening of banana bacterial endophytes for antagonistic activity against Fusarium oxysporum $\mathrm{f}$. sp. cubense on PDA employing spot-inoculation method: 2a, 3a and 4a showing inhibition zone correspond to Pseudomonas aeruginosa, Klebsiella variicola and Enterobacter cloacae, respectively. 


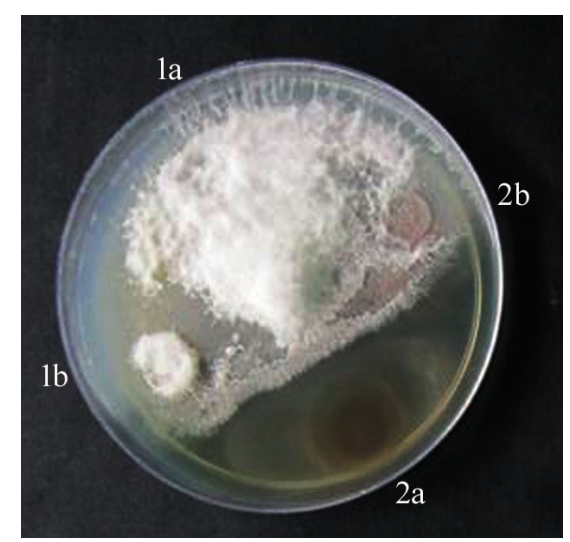

Figure 6. Confrontation assay between Fusarium oxysporum f. sp. cubense and the endophytic isolates GNS13.1a, 1b, 2a and 2b on NA + PDA 1:1 medium with GNS13.2a (Pseudomonas aeruginosa) displaying antagonistic activity against the pathogen.

\section{Discussion}

The present study was taken up with the objectives of isolating the bacterial endophytes associated with fieldderived banana suckers and evaluating them for the antagonistic activity against the Panama wilt pathogen, $F$. oxysporum f. sp. cubense. In continuation of the previous studies on banana which revealed the ubiquitous association of bacterial endophytes in the shoot-tips of cv. Grand Naine [7]-[9] and the intracellular colonization by the endophytic bacteria [10] [11], the current study confirms the association of diverse bacterial endophytes in the healthy field derived shoot-tips in banana suckers. The majority of bacterial isolates identified in this study belonged to Actinobacteria class which included organisms under 9 genera (Arthrobacter, Brevibacterium, Corynebacterium, Curtobacterium, Kocuria, Kytococcus, Micrococcus, Naumanella, Rothia, Tessaracoccus spp.) besides an unidentified bacterium. This is at variance from the earlier reports which indicated Proteobacteria as the predominant endophytes [8] [9]. In this study, Proteobacteria formed the second major class with Pseudomonas, Klebsiella and Enterobacter spp. from $\gamma$-subclass and Sphingomonas, Brevundimonas and Serratia spp. from $\alpha$-subclass. Bacillus and Staphylococcus spp. constituted the Firmicutes. Considerable variation from sucker to sucker in the CFU per unit tissue weight and the associated species has been observed. In an earlier study, the season of sucker-collection appeared to play a major role with more CFU and cultivable endophytes isolated during the rainy spell than summer months [7]. In the present study, no correlation was observed between the extent of colonization as indicated by $\mathrm{CFU} \mathrm{g}{ }^{-1}$ tissue and the number of isolates retrieved per sucker.

Diverse endophytic bacterial species have been isolated from banana in the previous studies which include Pantoea agglomerans, Citrobacter sp., Klebsiella pneumoniae [24], Cellulomonas fermentans, Acinetobacter lwoffii, Micrococcus luteus, Bacillus cereus, Paracoccus sp., Kocuria kristinae, Pseudacidovorax sp. [8], K. variicola, Enterobacter cloacae, Bacillus subtilis, Staphylococcus epidermidis, S. arlettae, Brevundimonas sp., Alcaligenes faecalis, Ralstonia mannitolilytica, Corynebacterium amycolatum, Pseudomonas stutzeri, Microbacterium testaceum, Methylobacterium hispanicum, Brachybacterium sp. and Brevibacterium sp. [9]. A series of root associated bacterial endophytes were isolated from cv. Prata Anã in Brazil which belonged to 10 genera (Agrobacterium, Aneurinibacillus, Bacillus, Enterobacter, Klebsiella, Lysinibacillus, Micrococcus, Paenibacillus, Rhizobium and Sporolactobacillus) constituting 15 species [25].

Endophytes, perhaps share a mutualistic association with the host [6] [26]. They draw the benefits of residency and nutritional support from the host. The host plant in turn is benefitted in many ways such as enhanced nutrient absorption leading to the improved vegetative growth and vigour of the host plant [3] [27]. Conferred resistance to plant pathogens is another beneficial effect which is accomplished through competitive root colonization, synthesis of bacterial allelochemicals and through induced systemic resistance [2] [4] [28].

Antagonism assays against fungi usually employ PDA or cornmeal agar on which the fungal culture and one or two test organisms are grown in a plate [29] [30]. This allows the testing of only one or two organisms per plate whereas spot-inoculation and pit-inoculation methods presented in this study permitted the testing of up to 12 organisms per plate. In this study, testing the 47 endophytic isolates for antagonistic activity against Foc 
showed three organisms displaying an inhibition effect towards the pathogen of which one organism $(P$. aeruginosa) exhibited good inhibition zone in confrontation assay too.

$F$. oxysporum f. sp. cubense is a devastating soil-borne pathogen which is very difficult to control. Biocontrol agents active against $F o c$ such as $P$. fluorescens have been reported in the earlier studies [18] [31]. P. aerugino$s a$ isolated from banana rhizosphere showed effective antagonistic activity towards $F o c$ and also displayed remarkable growth-promoting characteristics [32]. The identification of $P$. aeruginosa as an antagonist in this study opens the scope for engaging an endophytic organism as a potential biocontrol agent against Foc. Endophytes are able to colonize the plants internally and they share an intimate association with the host. The commonality of vascular niche colonization by the pathogen and endophytes offer great potential for the utilization of such antagonistic endophytes as biocontrol agents. Further efforts are now warranted towards testing this isolate as potential biocontrol agent against Foc.

Pseudomonas aeruginosa has been isolated as an endophyte from several other plant species and demonstrated as a biocontrol/growth promoting agent in different crop plants [33] [34]. P. aeruginosa is also known to be a human pathogen [34] [35]. However it is possible that the isolates associated with animal and plant systems are perhaps different. This aspect needs further investigations. The low proportion of endophytes with antagonistic activity against $F O C$ suggests the limited availability of such choice organisms. In the present study, $P$. aeruginosa was associated with only one of the 10 suckers explored. It would now be possible to introduce this organism in other suckers/plants through fortification at the time of field planting or at the hardening phase in the case of micropropagated plants. Banana being a long-duration crop of $10-12$ months, it would need more time and efforts to test the organisms as potential biocontrol agents and to evolve a biocontrol strategy which is in our next plan of action.

\section{Conclusion}

The present study has facilitated the isolation and identification of endophytic bacteria associated with the shoottip region in banana cv. Grand Naine yielding diverse organisms belonging to Actinobacteria, Proteobacteria and Firmicutes with Actinobacteria forming the commonest phylum. Testing the isolates for potential antagonistic activity against the banana wilt pathogen $F$. oxysporum f. sp. cubense brings out Pseudomonas aeruginosa as a promising antagonistic agent. Further studies are warranted to test the efficacy of this organism in biocontrol and the feasibility of introducing it in susceptible cultivars through inoculation of micropropagated plants or at field planting of suckers.

\section{Acknowledgements}

The study was funded partly under the ICAR-AMAAS Net-work project "Exploration of the endophytic microbial diversity in horticultural crops through metagenomics and cultivation". The supply of $F$. oxysporum $\mathrm{f}$. sp. cubense culture by Dr. T. R. Usharani, Division of Biotechnology, IIHR, is gratefully acknowledged. This publication bears IIHR contribution No. 16/2015.

\section{References}

[1] Mercado-Blanco, J. and Lugtenberg, B.J.J. (2014) Biotechnological Applications of Bacterial Endophytes. Current Biotechnology, 3, 60-75. http://dx.doi.org/10.2174/22115501113026660038

[2] Podolich, O., Ardanov, P., Zaets, I., Pirttilä, A.M. and Kozyrovska, N. (2015) Reviving of the Endophytic Bacterial Community as a Putative Mechanism of Plant Resistance. Plant and Soil, 388, 367-377. http://dx.doi.org/10.1007/s11104-014-2235-1

[3] Hardoim, P.R., van Overbeek, L.S. and Elsas, J.D.V. (2008) Properties of Bacterial Endophytes and Their Proposed Role in Plant Growth. Trends in Microbiology, 16, 463-471. http://dx.doi.org/10.1016/j.tim.2008.07.008

[4] Compant, S., Duffy, B., Nowak, J., Clement, C. and Barka, E.A. (2005) Use of Plant Growth-Promoting Bacteria for Biocontrol of Plant Diseases: Principles, Mechanism of Action and Future Prospects. Applied and Environmental Microbiology, 71, 4951-4959. http://dx.doi.org/10.1128/AEM.71.9.4951-4959.2005

[5] Azevedo, J.L., Maccheroni Jr., W., Pereira, J.O. and Luiz de Araújo, W. (2000) Endophytic Microorganisms: A Review on Insect Control and Recent Advances on Tropical Plants. Electronic Journal of Biotechnology, 3, 40-65. http://dx.doi.org/10.2225/vol3-issue1-fulltext-4

[6] Hallmann, J., Quadt-Hallmann, A., Mahaffe, W.F. and Kloepper, J.W. (1997) Bacterial Endophytes in Agricultural 
Crops. Canadian Journal of Microbiology, 43, 895-914. http://dx.doi.org/10.1139/m97-131

[7] Thomas, P., Swarna, G.K., Patil, P. and Rawal, R.D. (2008) Ubiquitous Presence of Normally Non-Cultivable Culturable Endophytic Bacteria in Field Shoot-Tips of Banana and Their Gradual Activation to Quiescent Cultivable Form in Tissue Cultures. Plant Cell Tissue and Organ Culture, 93, 39-54. http://dx.doi.org/10.1007/s11240-008-9340-x

[8] Thomas, P. and Soly, T.A. (2009) Endophytic Bacteria Associated with Growing Shoot Tips of Banana (Musa sp.) cv. Grand Naine and the Affinity of Endophytes to the Host. Microbial Ecology, 58, 952-964.

http://dx.doi.org/10.1007/s00248-009-9559-Z

[9] Thomas, P., Swarna, G.K., Roy, P.K. and Patil, P. (2008) Identification of Cultivable Culturable and Originally NonCulturable Endophytic Bacteria Isolated from Shoot Tip Cultures of Banana cv. Grand Naine. Plant Cell Tissue and Organ Culture, 93, 55-63. http://dx.doi.org/10.1007/s11240-008-9341-9

[10] Thomas, P. and Reddy, M.K. (2013) Microscopic Elucidation of Abundant Endophytic Bacteria Colonizing the Cell Wall-Plasma Membrane Peri-Space in the Shoot-Tip Tissue of Banana. AoB Plants, 5, Article ID: plt011. http://dx.doi.org/10.1093/aobpla/plt011

[11] Thomas, P. and Sekhar, A.C. (2014) Live Cell Imaging Reveals Extensive Intracellular Cytoplasmic Colonization of Banana by Normally Non-Cultivable Endophytic Bacteria. AoB PLANTS, 6, Article ID: plu002. http://dx.doi.org/10.1093/aobpla/plu002

[12] Stover, R.H. (1962) Fusarial Wilt (Panama Disease) of Bananas and Other Musa Species. Commonwealth Mycological Institute, Kew, England.

[13] Ploetz, R.C., Thomas, J.E. and Slabaugh, W.R. (2003) Diseases of Banana and Plantains. In: Ploetz, R.C., Ed., Diseases of Tropical Fruit Crops, CABI Publishing, CABI International, Wallingford, Oxon, 73-134. http://dx.doi.org/10.1079/9780851993904.0073

[14] Pérez-Vicente, L. and Dita, M.A. (2014) Fusarium Wilt of Banana or Panama Disease by Fusarium oxysporum f. sp. cubense: A Review on History, Symptoms, Biology, Epidemiology and Management. In: Pérez-Vicente, L., Dita, M.A. and Martínez-de la Parte, E., Eds., Technical Manual Prevention and Diagnostic of Fusarium Wilt (Panama Disease) of Banana Caused by Fusarium oxysporum f. sp. cubense Tropical Race 4 (TR4), FAO, Rome, 5-30.

[15] Bancroft, J. (1876) Report of the Board Appointed to Enquire into the Cause of Disease Affecting Livestock and Plants. Votes and Proceedings, 3, 1011-1038.

[16] Prasadji, J.K. and Smith, L. (2007) Fusarium Wilt of Banana: Cultivar Reaction, Race and Vegetative Compatibility Groups of Isolates of Fusarium oxysporum f. sp. cubense. In: Singh, H.P. and Uma, S., Eds., Banana: Technological Advancements, Association for the Improvement in Production and Utilization of Banana (AIPUB), C/o NRC for Banana, Trichy, 405-412.

[17] Ploetz, R.C. (1990) Variability in Fusarium oxysporum f. sp. cubense. Canadian Journal of Botany, 68, $1357-1363$. http://dx.doi.org/10.1139/b90-173

[18] Sivamani, E. and Gnanamanickam, S.S. (1988) Biological Control of Fusarium oxysporum f. sp. cubense in Banana by Inoculation with Pseudomonas fluorescens. Plant and Soil, 107, 3-9. http://dx.doi.org/10.1007/BF02371537

[19] Thangavelu, R., Kumar, K.M., Ganga Devi, P. and Mustaffa, M.M. (2011) Genetic Diversity of Fusarium oxysporum f. sp. cubense isolatees (Foc) of India by Inter Simple Sequence Repeats (ISSR) Analysis. Molecular Biotechnology, 51, 203-211. http://dx.doi.org/10.1007/s12033-011-9457-8

[20] Sun, J.B., Peng, M., Wang, Y.G., Zhao, P.J. and Xia, Q.Y. (2011) Isolation and Characterization of Antagonistic Bacteria against Fusarium Wilt and Induction of Defense Related Enzymes in Banana. African Journal of Microbiology Research, 5, 509-515.

[21] Ho, Y.N., Chiang, H.M., Chao, C.P., Su, C.C., Hsu, H.F., Guo, C., Hsieh, J.L. and Huang, C.C. (2015) In Planta Biocontrol of Soilborne Fusarium Wilt of Banana through a Plant Endophytic Bacterium, Burkholderia cenocepacia 869T2. Plant and Soil, 387, 295-306. http://dx.doi.org/10.1007/s11104-014-2297-0

[22] Thomas, P., Sekhar, A.C. and Mujawar, M.M. (2012) Non-Recovery of Varying Proportions of Viable Bacteria during Spread-Plating Governed by the Extent of Spreader Usage and Proposal for an Alternate Spotting-Spreading Approach to Maximize the CFU. Journal of Applied Microbiology, 113, 339-350. http://dx.doi.org/10.1111/j.1365-2672.2012.05327.x

[23] White, T.J., Bruns, T., Lee, S. and Taylor, J. (1990) Amplification and Direct Sequencing of Fungal Ribosomal RNA Genes for Phylogenetics. In: Innis, M.A., Gelfand, D.H., Shinsky, J.J. and White, T.J., Eds., PCR Protocols: A Guide to Methods and Applications, Academic Press, Inc., New York, 315-322. http://dx.doi.org/10.1016/B978-0-12-372180-8.50042-1

[24] Martinez, L., Caballero-Mellado, J., Orozco, J. and Martinez-Romero, E. (2003) Diazotrophic Bacteria Associated with Banana (Musa spp.). Plant and Soil, 257, 35-47. http://dx.doi.org/10.1023/A:1026283311770

[25] Souza, S.A., Xavier, A.A., Costa, M.R., Cardoso, A.M.S., Pereira, M.C.T. and Nietsche, S. (2013) Endophytic Bacte- 
rial Diversity in Banana "Prata Anã" (Musa spp.) Roots. Genetics and Molecular Biology, 36, 252-264. http://dx.doi.org/10.1590/S1415-47572013000200016

[26] Rosenblueth, M. and Martinez-Romero, E. (2006) Bacterial Endophytes and Their Interaction with Hosts. Molecular Plant Microbe Interactions, 19, 827-837. http://dx.doi.org/10.1094/MPMI-19-0827

[27] Ting, A.S.Y., Sariah, M., Jugah, K., Son, R. and Gurmit, S. (2008) Endophytic Microorganisms as Potential Growth Promoters of Banana. BioControl, 53, 541-553. http://dx.doi.org/10.1007/s10526-007-9093-1

[28] Ting, A.S.Y., Meon, S., Kadir, J., Radu, S. and Singh, G. (2010) Induction of Host Defence Enzymes by the Endophytic Bacterium Serratia marcescens, in Banana Plantlets. International Journal of Pest Management, 56, 183-188. http://dx.doi.org/10.1080/09670870903324198

[29] Chernin, L., Ismailov, Z., Haran, S. and Chet, I. (1995) Chitinolytic Enterobacter agglomerans Antagonistic to Fungal Plant Pathogens. Applied and Environmental Microbiology, 61, 1720-1726.

[30] Crawford, D.L., Lynch, J.M., Whipps, J.M. and Ousley, M.A. (1993) Isolation and Characterization of Actinomycete Antagonists of a Fungal Root Pathogen. Applied and Environmental Microbiology, 59, 3899-3905.

[31] Sun, J.B., Peng, M., Wang, Y.G., Zhao, P.J. and Xia, Q.Y. (2011) Isolation and Characterization of Antagonistic Bacteria against Fusarium Wilt and Induction of Defense Related Enzymes in Banana. African Journal of Microbiology Research, 5, 509-515.

[32] Ayyadurai, N., Ravindra Naik, P., Sreehari Rao, M., Sunish Kumar, R., Samrat, S.K., Manohar, M. and Sakthivel, N. (2006) Isolation and Characterization of a Novel Banana Rhizosphere Bacterium as Fungal Antagonist and Microbial Adjuvant in Micropropagation of Banana. Journal of Applied Microbiology, 100, 926-937. http://dx.doi.org/10.1111/j.1365-2672.2006.02863.x

[33] Gupta, G., Panwar, J. and Jha, P.N. (2013) Natural Occurrence of Pseudomonas aeruginosa, a Dominant Cultivable Diazotrophic Endophytic Bacterium Colonizing Pennisetum glaucum (L.) R. Br. Applied Soil Ecology, 64, $252-261$. http://dx.doi.org/10.1016/j.apsoil.2012.12.016

[34] Kumar, A., Munder, A., Aravind, R., Eapen, S.J., Tummler, B. and Raaijmakers, J.M. (2013) Friend or Foe: Genetic and Functional Characterization of Plant Endophytic Pseudomonas aeruginosa. Environmental Microbiology, 15, $764-$ 779. http://dx.doi.org/10.1111/1462-2920.12031

[35] Pukatzki, S., Kessin, R.H. and Mekalanos, J.J. (2002) The Human Pathogen Pseudomonas aeruginosa Utilizes Conserved Virulence Pathways to Infect the Social Amoeba Dictyostelium discoideum. Proceedings of the National Academy of Sciences, 99, 3159-3164. http://dx.doi.org/10.1073/pnas.052704399 